\title{
Fresnel Dragging Explained by a Classical Model of Light Transmission in Optical Media
}

\author{
Declan Traill \\ Melbourne, Australia \\ Email:declan@netspace.net.au
}

How to cite this paper: Traill, D. (2021) Fresnel Dragging Explained by a Classical Model of Light Transmission in Optical Media. Journal of Applied Mathematics and Physics, 9, 1883-1889. https://doi.org/10.4236/jamp.2021.98121

Received: June 27, 2021

Accepted: August 8, 2021

Published: August 11, 2021

Copyright $\odot 2021$ by author(s) and Scientific Research Publishing Inc. This work is licensed under the Creative Commons Attribution International License (CC BY 4.0).

http://creativecommons.org/licenses/by/4.0/

\section{(c) (i) Open Access}

\begin{abstract}
The original mathematical treatment used in the analysis of the Fizeau experiment of 1851, which measured the relative speed of light in a moving medium, assumes that light travels through the water in a smooth continuous flow, at a speed less than the speed of light in a vacuum (relative to the water). Thus, it assumes that the water's velocity vector can simply be added to that of the light. However, light is transmitted through optical media, such as water, by a continuous process of charge excitation (semi-absorption) and re-emission by the water molecules; but travels between them at the full speed of light (in a vacuum). Thus, the mathematics describing the process of Fresnel dragging must be formulated differently and can then be explained by classical physics, allowing the entire process to be fully visualized.
\end{abstract}

\section{Keywords}

Fresnel, Dragging, Optical, Fizeau, Absorption, Emission, Delay, Refractive, Index, Relativity, Classical, Physics

\section{Introduction}

Fresnel's formula describing the effect on the transmission speed of light travelling through a moving optical medium was originally formulated in 1818, and based on the idea that the ether (which light was presumed to travel through at the time) was partially dragged along by the moving medium; as discussed in [1].

Later in 1851, Hippolyte Fizeau conducted an experiment to test this prediction and found it to be true [2]. It was again tested and confirmed by Michelson and Morley in 1886 [3]; the cause of the effect is still interpreted as entrained ether.

In 1907 soon after Relativity emerged as a new theory (in 1905), Max Laue ex- 
plained the Fresnel dragging effect in terms of Relativistic addition of velocities and Einstein soon adopted this approach (see [1]).

These theories explaining the Fresnel dragging effect make the same assumption that the light travels at a smooth, continuous rate determined by the refractive index of the optical medium through which it is travelling. However, the transmission of light through an optical medium occurs on the microscopic level by a process of absorption/emission due to the light's electromagnetic field, causing the charges comprising the optical medium to oscillate and introduce a phase delay in the re-emitted light [4].

If this is taken into account, then the explanation for Fresnel dragging can no longer assume a smooth, continuous rate of flow of light through the medium, but would be described by a stop-start process. In such an explanation, the molecules of the optical medium would slow the light's progress, while their charges oscillate, before retransmitting the light after a short time delay. The light would travel unimpeded (at the full speed of light in a vacuum) between the molecules.

It should be noted here that the optical process at work when the light passes through the water is really that the propagating light wave is actually the sum of the original light wave and of the light waves emitted by the charges which have been set into oscillation by the original light wave. The resulting electromagnetic wave sum would vary in propagation speed with distance from the oscillating charges in the water molecules, but from a modeling perspective and from a Theoretical Physics point of view, this can be simplified into two distinct stages of propagation: 1) The light travels at the water's speed $\mathrm{v}$ whilst absorbed by the water as the energy of the oscillating charges. 2) The light moving at full speed of light $\mathrm{c}$ when traveling in the vacuum between water molecules.

There have been some attempts to explain Fresnel dragging using a classical physics approach based on frequency change or perturbation (see [5] [6] [7] [8]), but none show exactly how the Fresnel dragging coefficient is derived from the principles of the model. Very little attention seems to have been given to the possibility of a classical model for Fresnel Dragging.

Here I present a detailed explanation and mathematical model by which Fresnel's dragging coefficient can be derived, and thus the process of Fresnel dragging is completely explained using classical physics principles alone. Therefore, there it is possible to visualize exactly what is happening at every step of the process and there is no need to appeal to a Relativistic approach which tends to obscure the real reasons for the phenomenon. Also, other works [5] [6] [7] [8] that claim to explain the effect of Fresnel Dragging based on frequency change or perturbation lack the clarity that allows the reader to see and actually understand exactly what is happening at the microscopic level to cause the Fresnel Dragging effect. I think that every aspect of Physics should be able to be visualized and understood in terms of simple Classical Physics principles, as I have done here. It is only when we can do this that we can truly understand what is going on and be confident that we are not fooling ourselves with mathematics or abstract theoretical concepts. 


\section{The New Classical Model}

The refractive index of water is represented by $n$. This number is the factor by which light's progress is delayed when travelling through stationary water. It represents two properties of the water.

1) The number of water molecules encountered per second by light as it travels through the water.

2) The amount of time by which light is delayed by the processes of absorption and re-emission when it encounters water molecules.

Light's speed when slowed by the refractive index $n$ can be defined as:

$$
c_{n}=\frac{c}{n}
$$

For a tube of stationary water, the time taken for light to travel through a tube of length $L$ is simply:

$$
\Delta t=\frac{L}{c_{n}}
$$

For light travelling through moving water, such as in the Fizeau experiment (see [2]) where light is passing along the length of a tube through which water is flowing rapidly, the calculation is more complex.

While the energy of the light is absorbed by a water molecule, and until it is re-emitted as light again, it will be carried downstream (or upstream) by the water, at the water's speed $v$. Thus, depending if the light is travelling upstream or downstream through the flowing water, it will be carried backwards or forwards along its travel path by the water.

This will have the effect of extending or reducing the optical path length that the light must travel to reach the other end of the tube in the experiment. The proportion of the total travel time that the water molecules carry the energy of the light with them is the difference between the water's refractive index and that of a vacuum as a fraction of the water's refractive index $n$. Therefore, the fraction representing this amount is:

$$
\frac{n-1}{n}=1-\frac{1}{n}
$$

If the length of the tube is $L$ and the time taken by light to travel from one end of the tube to the other is $\Delta t_{u p}$ or $\Delta t_{\text {down }}$, then the time taken by the light to travel up/down the tube will be the total optical path length when travelling upstream/downstream divided by its speed $c_{n}$.

For light travelling upstream:

$$
\Delta t_{u p}=\frac{L+\left(1-\frac{1}{n}\right) v \Delta t_{u p}}{c_{n}}
$$

For light travelling downstream:

$$
\Delta t_{\text {down }}=\frac{L-\left(1-\frac{1}{n}\right) v \Delta t_{\text {down }}}{c_{n}}
$$


However, there is an additional effect that occurs simultaneously due to the flow of the water. As the water is flowing towards the light in the upstream direction, and away from the light in the downstream direction, the number of molecules of water encountered by the light per second of its travel will either be higher or lower than the number encountered if light was moving through stationary water. This will also act to increase or decrease the travel time of the light.

Light travels between water molecules at the speed of light in a vacuum, represented by the symbol $c$. Other than the delay whilst light is absorbed by the water molecules, the number of water molecules the light encounters each second when travelling through stationary water is determined by this speed and the density of the water. If the density of the water molecules is $\rho$ (in units of molecules per meter) then an expression for the number of water molecules encountered by light each second is $c \rho$. When the water is moving, the additional rate at which water molecules are moving towards or away from the light is determined by the water's speed $v$. Over the whole travel time upstream or downstream, the amount of additional water molecules encountered by the light will be proportional to $v$ multiplied by $\Delta t_{u p}$ or $\Delta t_{\text {down }}$. Thus, again using the density of the water, the number of extra water molecules encountered would be $v \Delta t_{\text {up }} \rho$ or $v \Delta t_{\text {down }} \rho$. These amounts expressed as a fraction of the number of water molecules encountered by light travelling through stationary water each second are:

$$
\begin{gathered}
\frac{v \Delta t_{u p} \rho}{c \rho}=\frac{v \Delta t_{u p}}{c} \\
\frac{v \Delta t_{\text {down }} \rho}{c \rho}=\frac{v \Delta t_{\text {down }}}{c}
\end{gathered}
$$

However, as it is the water that is moving (towards, or receding from the light) rather than the light moving that causes this additional effect, there is no additional space being travelled by the light due to the water's motion. To determine the extra delay time experienced by the light due to these additional water molecules, Equations (6) and (7) must be multiplied by the proportion of time that the light interacts with just the molecules of water, given by Equation (3). Thus, they become:

$$
\begin{gathered}
\frac{\left(1-\frac{1}{n}\right) v \Delta t_{u p}}{c} \\
\frac{\left(1-\frac{1}{n}\right) v \Delta t_{\text {down }}}{c}
\end{gathered}
$$

Then, so as to express these times with a denominator of $c_{n}$ rather than $c$, both the numerator and denominator are divided by $n$, and using Equation (1), giving: 


$$
\begin{gathered}
\frac{\frac{1}{n}\left(1-\frac{1}{n}\right) v \Delta t_{u p}}{c_{n}} \\
\frac{\frac{1}{n}\left(1-\frac{1}{n}\right) v \Delta t_{\text {down }}}{c_{n}}
\end{gathered}
$$

So, when these additional delays due to the water's motion are added to Equations (4) and (5), the total travel times are as follows.

For light travelling upstream:

$$
\Delta t_{u p}=\frac{L+\left(1-\frac{1}{n}\right) v \Delta t_{\text {up }}+\frac{1}{n}\left(1-\frac{1}{n}\right) v \Delta t_{\text {up }}}{c_{n}}
$$

Simplifying gives:

$$
\begin{gathered}
\Delta t_{u p}=\frac{L+\left(1+\frac{1}{n}\right)\left(1-\frac{1}{n}\right) v \Delta t_{u p}}{c_{n}} \\
\Delta t_{u p}=\frac{L+\left(1-\frac{1}{n^{2}}\right) v \Delta t_{u p}}{c_{n}}
\end{gathered}
$$

Solving for $\Delta t_{u p}$ gives:

$$
\Delta t_{u p}=\frac{L}{c_{n}-v\left(1-\frac{1}{n^{2}}\right)}
$$

Similarly, for light travelling downstream:

$$
\Delta t_{\text {down }}=\frac{L-\left(1-\frac{1}{n}\right) v \Delta t_{\text {down }}-\frac{1}{n}\left(1-\frac{1}{n}\right) v \Delta t_{\text {down }}}{c_{n}}
$$

Simplifying gives:

$$
\begin{gathered}
\Delta t_{\text {down }}=\frac{L-\left(1+\frac{1}{n}\right)\left(1-\frac{1}{n}\right) v \Delta t_{\text {down }}}{c_{n}} \\
\Delta t_{\text {down }}=\frac{L-\left(1-\frac{1}{n^{2}}\right) v \Delta t_{\text {down }}}{c_{n}}
\end{gathered}
$$

Solving for $\Delta t_{\text {down }}$ gives:

$$
\Delta t_{\text {down }}=\frac{L}{c_{n}+v\left(1-\frac{1}{n^{2}}\right)}
$$

\section{Verification of the New Model}

In the original analysis and mathematics for the Fizeau experiment [2], where 
the assumption is that the light and water velocities can simply be added, the following upstream and downstream times were calculated:

$$
\begin{gathered}
t_{u p}=\frac{L}{C_{n}-v^{\prime}} \\
t_{\text {down }}=\frac{L}{C_{n}+v^{\prime}}
\end{gathered}
$$

where $v^{\prime}$ is the water's speed multiplied by the Fresnel dragging coefficient, required to explain the result of the experiment:

$$
v^{\prime}=v\left(1-\frac{1}{n^{2}}\right)
$$

Substituting Equation (22) into my derived Equations (15) and (19) gives Equations (20) and (21), thus showing that my new model gives the correct result.

\section{Conclusion}

Treating the passage of light through optical media as a process of continual absorption and emission allows the phenomenon known as Fresnel dragging to be explained by Classical Physics. The new mathematical formulation models light as being delayed by molecules and traveling at their speed for a short time, but then travelling at the full speed of light in the vacuum between the molecules, rather than as a smooth continuous flow of light through the water at a slower speed. This new explanation allows one to be able to fully visualize the processes at work that lead to the phenomenon of Fresnel Dragging, in a way that previous explanations have not been able to do. Based on this theory, the process of Fresnel Dragging occurring in the Fizeau experiment has been successfully modelled. The results of this modelling can be seen in short video clips available here [9].

\section{Conflicts of Interest}

The author declares no conflicts of interest regarding the publication of this paper.

\section{References}

[1] Galina, W. (2012) Albert Einstein and the Fizeau 1851 Water Tube Experiment. Cornell University Library, Cornell, 1. https://arxiv.org/pdf/1204.3390

[2] Fizeau, M.H. (1860) On the Effect of the Motion of a Body upon the Velocity with Which It Is Traversed by Light. The London, Edinburgh and Dublin Philosophical Magazine and Journal of Science, 251, 245-260. http://www.relativitycalculator.com/images/fizeau fresnel experiment/fizeau.pdf https://doi.org/10.1080/14786446008642856

[3] Michelson, A.A. and Morley, E.W. (1886) Influence of Motion of the Medium on the Velocity of Light. American Journal of Science, 31, 377-386.

https://en.wikisource.org/wiki/Influence of Motion of the Medium on the Velo city of Light 
https://doi.org/10.2475/ajs.s3-31.185.377

[4] Refractive Index. Wikipedia. https://en.wikipedia.org/wiki/Refractive index

[5] Sokolov, G.V. (2013) The Fizeau Experiment with Moving Water. The General Science Journal, 1-8.

http://www.gsjournal.net/Science-Journals/Research\%20Papers-Mechanics\%20/\%2 0Electrodynamics/Download/5014

[6] Gennadiy and Vitali (2017) Optical Fizeau Experiment with Moving Water Is Explained without Fresnel's Hypothesis and Contradicts Special Relativity. Journal of Physical Mathematics, 8, 1.

https://www.hilarispublisher.com/open-access/optical-fizeau-experiment-with-mov ing-water-is-explained-withoutfresnels-hypothesis-and-contradicts-special-relativit y-2090-0902-1000207.pdf

[7] Drezet, A. (2005) The Physical Origin of the Fresnel Drag of Light by a Moving Dielectric Medium. The European Physical Journal B: Condensed Matter and Complex Systems, 45, 103-110. https://arxiv.org/pdf/physics/0506004v1.pdf https://doi.org/10.1140/epjb/e2005-00169-4

[8] Cahill, R.T. and Brotherton, D. (2011) Experimental Investigation of the Fresnel Drag Effect in RF Coaxial Cables. Progress in Physics, 1, 43-48. http://www.ptep-online.com/2011/PP-24-04.PDF

[9] Traill, D.A. (2021) Energy Field Theory-Demos-Fresnel Dragging. https://www.energyfieldtheory.com/fresnel-dragging 